\title{
Electrochemical SERS at a structured gold surface
}

\author{
Mamdouh E. Abdelsalam ${ }^{a}$, Philip N. Bartlett ${ }^{\mathrm{a}, *}$, Jeremy J. Baumberg ${ }^{\mathrm{b}}$, \\ Suzanne Cintra ${ }^{\text {a }}$, Tim A. Kelf ${ }^{b}$, Andrea E. Russell ${ }^{\text {*a }}$ \\ ${ }^{\text {a }}$ School of Chemistry, University of Southampton, Southampton SO17 1 BJ, UK \\ ${ }^{\mathrm{b}}$ School of Physics and Astronomy, University of Southampton, Southampton SO17 1BJ, UK
}

Received 5 April 2005; received in revised form 22 April 2005; accepted 22 April 2005

Available online 2 June 2005

\begin{abstract}
Templated electrodeposition of gold to produce thin $(<1 \mu \mathrm{m})$ films containing a close packed hexagonal array of uniform sphere segment voids is shown to give surfaces which show strong surface enhancement for Raman scattering from molecules adsorbed at the surface. The magnitude of this is enhancement is determined by the precise geometry of the surface and depends on the choice of void diameter and film thickness. The resulting SER active surfaces are stable, reusable, give reproducible surface enhancement and can be used for in situ electrochemical SERS studies.
\end{abstract}

(C) 2005 Elsevier B.V. All rights reserved.

Keywords: Templated electrodeposition; Nanostructured gold; Electrochemical SERS; Pyridine adsorption

\section{Introduction}

In the 30 years since the initial observation of Raman spectra for pyridine adsorbed on roughened silver electrodes [1] and the subsequent realisation that there was a very significant $\left(\sim 10^{6}\right)$ surface enhancement of the Raman intensity at the roughened silver surface $[2,3]$ the technique of surface enhanced Raman spectroscopy has been widely applied to study molecules at electrochemically roughened $\mathrm{Cu}, \mathrm{Ag}$ and $\mathrm{Au}$ electrode surfaces [4-6]. SERS has a number of attributes which make it a very attractive in situ technique for the study of electrode surfaces and electrochemical processes. Firstly, the surface enhancement is highly surface selective so the technique is sensitive to molecules adsorbed at, or very close to, the electrode surface and it thus discriminates against molecules in the bulk solution. Secondly,

\footnotetext{
${ }^{*}$ Corresponding author. Tel.: +44 2380 592372/06; fax: +44 2380 593781.

E-mail addresses: pnb@soton.ac.uk (P.N. Bartlett), a.e.russell@ soton.ac.uk (A.E. Russell).
}

SERS gives information about the molecular structure of the adsorbate, its orientation at the surface [7,8] and the dependence of orientation and coverage on applied potential. Finally, the Raman cross section for water is low so that SERS can be easily used to study electrodes in aqueous solution.

Despite these obvious advantages, SERS also suffers significant limitations. Although the electrochemical roughening process generates SERS active surfaces for $\mathrm{Cu}, \mathrm{Ag}$, and $\mathrm{Au}$ the degree of surface enhancement is not reproducible, varies from place to place across the electrode surface and is not stable. In addition, the electrochemical roughening process largely restricts the use of the electrodes to aqueous solutions containing chloride and the roughened surfaces suffer irreversible loss of enhancement at high negative potentials. Consequently a number of alternative SERS substrates have been proposed over the past 30 years. These include the use of grating structures [9], colloidal particle arrays [10], and geometrically structured metal surfaces $[7,8]$ with more or less success. Not all of these are suitable for electrochemical SERS measurements and there 
remains a clear need for SER active electrodes which are robust, easy to fabricate and give reproducible surface enhancement.

It is generally accepted that there are two contributions to the surface enhancement [11]; a charge transfer (CT) enhancement [12] arising from chemisorption of the adsorbate at the metal surface and an electromagnetic (EM) enhancement [13]. Of these two the EM contribution is usually the more significant and is not adsorbate specific. The EM enhancement arises from the focusing of the electric field at certain places on the metal surface and is therefore strongly dependent on the surface morphology and precise shape of the roughness features at the metal surface.

In a separate paper, we showed that electrodeposition through monolayer colloidal templates can be used to produce SERS active silver surfaces [14]. In this paper, we extend this work to show that electrodeposition through monolayer colloidal templates can be used to produce highly SERS active gold surfaces for which the surface enhancement is reproducible across the sample. We show that the structured gold surfaces are stable and reusable, that enhancement depends upon the precise structure of the gold surface, requiring the correct choice of template sphere diameter and film thickness to maximise the effect. Finally, we report the first example of the use of these surfaces for electrochemical SERS.

\section{Experimental}

Evaporated gold electrodes used as substrates were prepared by depositing $10 \mathrm{~nm}$ of chromium, followed by $200 \mathrm{~nm}$ of gold onto $1 \mathrm{~mm}$ thick glass microscope slides. These gold substrates were thoroughly cleaned before use by sonication in deionized water for $30 \mathrm{~min}$, sonication in isopropanol for $90 \mathrm{~min}$, then rinsed with deionized water and dried under a stream of argon (BOC Gases). Cysteamine was self-assembled onto the gold electrodes by immersing the freshly cleaned substrate in a $10 \mathrm{mmol} \mathrm{dm}^{-3}$ ethanolic solution of cysteamine at room temperature for several days. Under these conditions the $\mathrm{SH}$ group of the thiol molecule readily reacts with the gold creating a dense monolayer [15]. Modifying the gold substrate with cysteamine has two effects. First, it increases the efficiency of the assembly of the polystyrene spheres on the gold substrate by increasing the substrate-particle interaction. This is because the substrate surface is positively charged as a results of the chemical modification with the cysteamine and the spheres have a negative surface charge due to the sulfate groups used to stabilize the polystyrene suspension [16]. Second, it increases the wettability of the gold substrate - the contact angle for water is decreased from $70^{\circ}$ to $40^{\circ}$ by treating with cysteamine. This signif- icant increase in the wettabily assists the formation of a thin water film between the spheres and the substrate at the meniscus and this thin water film produces a substantial force (the capillary immersion force) that directs the spheres to assemble in a well ordered and close packed structure [17].

Templates were made from monodisperse polystyrene latex spheres (Duke Scientific Corporation, $1 \mathrm{wt} \%$ solution in water, coefficient of variation in diameter $1.3 \%$ ). Assembly of the particles was carried out in a thin layer cell (more details can be found in an earlier publication [18]). Gold was deposited from a cyanide free gold plating solution containing $7.07 \mathrm{~g} \mathrm{dm}^{-3}$ gold (Tech. Gold 25, Technic Inc. Cranston, RI) using a conventional three-electrode cell controlled by an Autolab PGSTAT30 under potentiostatic conditions at $-0.7 \mathrm{~V}$ vs. SCE and $25^{\circ} \mathrm{C}$. After deposition the samples were soaked in THF for two hours to remove the polystyrene template. A Philips XL30 ESEM was used to image both the polystyrene templates and the macroporous metal films. All Raman spectra were recorded on a Renishaw Raman 2000 system using a $633 \mathrm{~nm} \mathrm{HeNe}$ laser with $5 \mu \mathrm{m}$ diameter spot size and $3 \mathrm{~mW}$ power using a single $10 \mathrm{~s}$ accumulation unless otherwise stated. Benzene thiol was adsorbed onto the gold surface by soaking in a $5 \mathrm{mM}$ solution in ethanol for $30 \mathrm{~min}$. The samples were then rinsed with ethanol, and left to dry in air for 15 min before measurement. Electrochemically roughened gold surfaces used for comparison were prepared by cycling the potential of a gold plate in $0.1 \mathrm{M}$ $\mathrm{KCl}$ following the procedure described by Tian [8]. Briefly this involves 25 cycles of cycling from -0.3 to $1.2 \mathrm{~V}$ vs. SCE at $1 \mathrm{~V} \mathrm{~s}^{-1}$, holding the potential at $1.2 \mathrm{~V}$ for $30 \mathrm{~s}$, sweeping back to $-0.3 \mathrm{~V}$ at $0.5 \mathrm{~V} \mathrm{~s}^{-1}$, then holding the potential at $-0.3 \mathrm{~V}$ for $1.2 \mathrm{~s}$. At the end of this treatment the roughened gold surface appears dark brown.

\section{Results and discussion}

Structured gold surfaces were prepared by electrodeposition through close packed monolayers of polystyrene spheres with diameters between 450 and $900 \mathrm{~nm}$ assembled onto evaporated gold electrodes. The thickness of the electrodeposited film was controlled by varying the charge passed and was never more that about $80 \%$ of the template diameter. After deposition the polystyrene was removed to leave the thin structured gold films containing a regular hexagonal array of uniform segment sphere voids. These surfaces are strongly coloured, the precise colour depending on the viewing angle, void diameter and film thickness [18]. Fig. 1 shows SEM images of structured gold surfaces and an electrochemically roughened gold surface. The structured gold films have different thicknesses and were produced by 

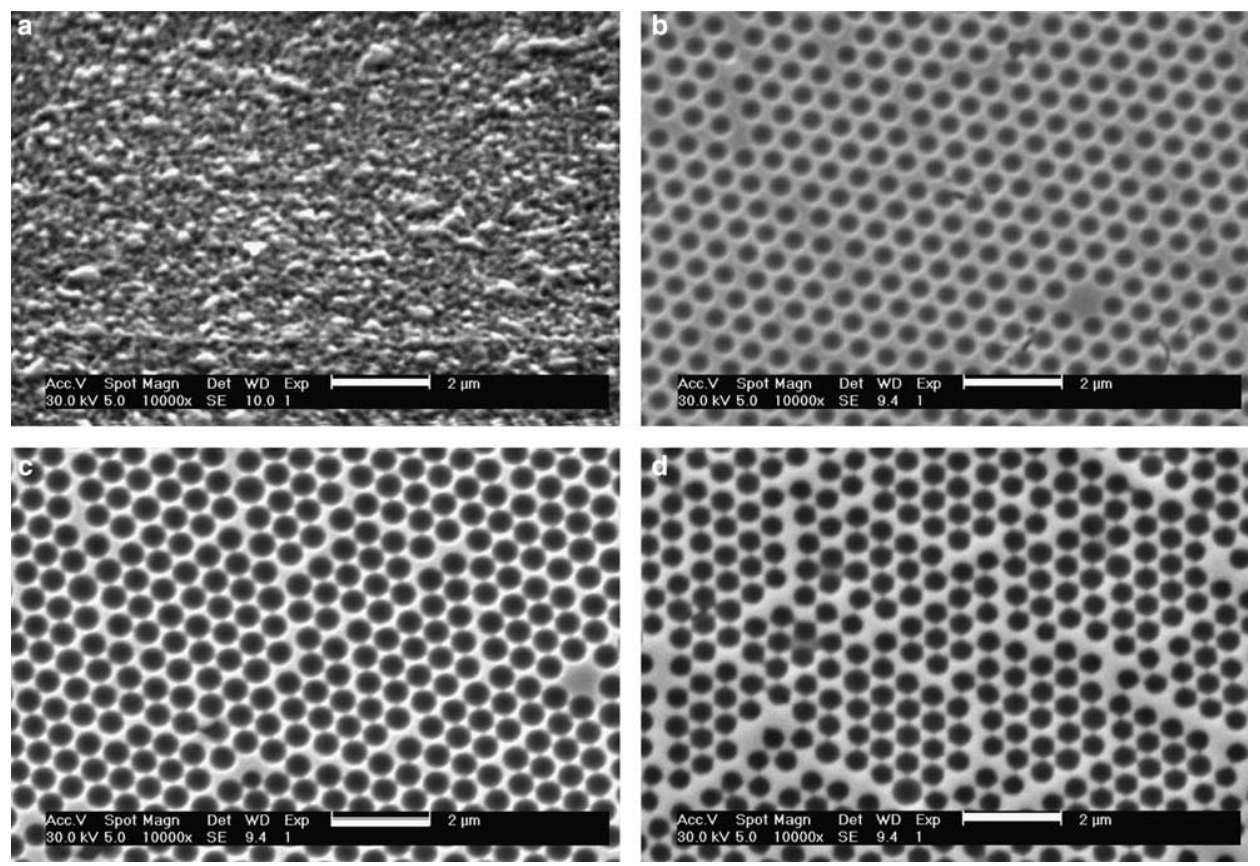

Fig. 1. SEM images of: (a) an electrochemically roughened gold surface and (b,c) structured gold surfaces. The structured gold surfaces were produced using $600 \mathrm{~nm}$ diameter template spheres and have thickness of (a) $150 \mathrm{~nm}$, (b) $260 \mathrm{~nm}$ and (c) $460 \mathrm{~nm}$, respectively. The scale bar is $2 \mu \mathrm{m}$ in each case.

electrochemical deposition through templates consisting of a monolayer of $600 \mathrm{~nm}$ diameter polystyrene spheres [18]. The mouths of the pores are nearly circular and their diameter changes with the film thickness. The regular array of pores in the structured gold surface is clearly visible whereas the roughened surface shows a random distribution of sub-micron features. Note that the structured gold surface is not itself very rough; electrochemical measurements of the surface area of these structured surfaces using oxide stripping in acidic solution shows an increase in surface area of a factor of 1.6 over the projected geometric area for the $450 \mathrm{~nm}$ thick sample. This increase is consistent with a smooth, yet sculpted, gold surface and is agrees with the expected increase in area due to the presence of the segment sphere voids.

Fig. 2 shows a comparison of SERS spectra recorded for benzene thiol at a structured gold surface and at an electrochemically roughened gold surface. The intensity of the SERS signal for the roughened gold surface varies significantly (by a factor of $\sim 10$ ) from place to place across the sample [19], the spectrum shown corresponds to the largest enhancement found. These SERS spectra for benzene thiol on gold agree well with those in the literature in terms of the band positions and relative intensities [20,21]. The assignment of SERS peaks for the adsorbed benzene thiol is given in Table 1. From Fig. 2 , it is clear that the enhancement at the structured gold surface is considerably larger than the best enhancement found at the roughened surface. In control experiments, only extremely weak SERS spectra (indistinguishable

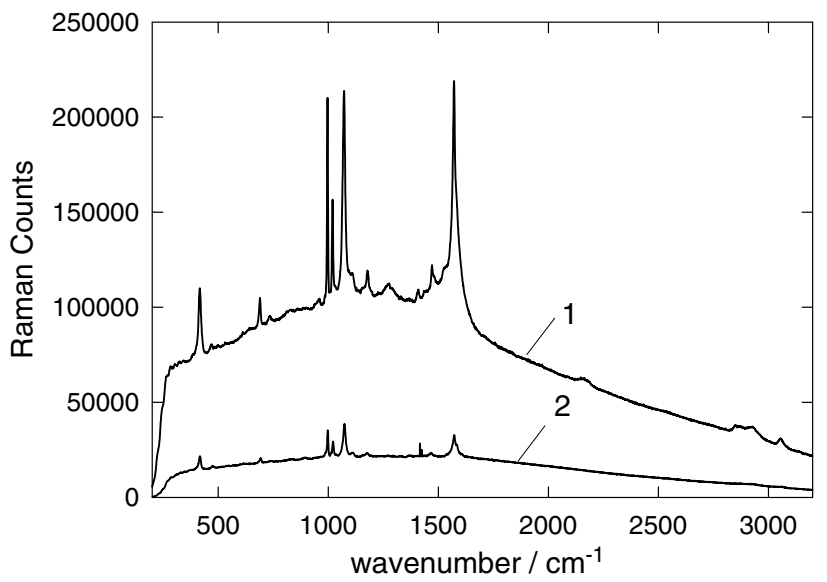

Fig. 2. SER spectra for benzene thiol adsorbed on: (1) a structured gold surface and (2) electrochemically roughened gold surface. The structured gold surface was produced using $600 \mathrm{~nm}$ diameter template spheres and is $150 \mathrm{~nm}$ thick. Spectra were obtained with a $633 \mathrm{~nm}$ HeNe laser using $3 \mathrm{~mW}$ power and a single $10 \mathrm{~s}$ accumulation with a $5 \mu \mathrm{m}$ spot size.

from baseline on the scale of Fig. 2) were found for benzene thiol on the evaporated gold substrate or for nonstructured gold films electrodeposited on the evaporated gold substrate. The surface enhancement on the structured gold surface is reproducible from place to place across the surface. The reproducibility of the surface enhancement was assessed by recording 50 spectra at different places on the structured gold surface at nominally the same film thickness. A standard deviation of $10 \%$ was found in the height of the peak at $1021 \mathrm{~cm}^{-1}$. 
Table 1

Assignment of the SERS peaks for benzene thiol on structured gold

\begin{tabular}{ll}
\hline SERS peak $\left(\mathrm{cm}^{-1}\right)$ & Assignment ${ }^{\mathrm{a}}$ \\
\hline 3055 & $\mathrm{a}_{1}, v(\mathrm{C}-\mathrm{H})$ \\
1571 & $\mathrm{a}_{1}, v(\mathrm{C}-\mathrm{C}-\mathrm{C})$ \\
1471 & $\mathrm{a}_{1}, v(\mathrm{C}-\mathrm{C})$ \\
1177 & $\mathrm{a}_{1}, v(\mathrm{C}-\mathrm{H})$ \\
1071 & $\mathrm{a}_{1}, v(\mathrm{C}-\mathrm{C}-\mathrm{C})$ and $v(\mathrm{C}-\mathrm{S})$ \\
1021 & $\mathrm{a}_{1}, v(\mathrm{C}-\mathrm{H})$ \\
996 & $\mathrm{a}_{1}, v(\mathrm{C}-\mathrm{C}-\mathrm{C})$ \\
689 & $\mathrm{a}_{1}, v(\mathrm{C}-\mathrm{C}-\mathrm{C})$ and $v(\mathrm{C}-\mathrm{S})$ \\
416 & $\mathrm{a}_{1}, v(\mathrm{C}-\mathrm{C}-\mathrm{C})$ and $v(\mathrm{C}-\mathrm{S})$ \\
\hline
\end{tabular}

a Assigned on the basis of Han et al. [26].

When comparing different structured gold films prepared under the same conditions and with nominally the same thickness a standard deviation of $13 \%$ was found for the peak at $1021 \mathrm{~cm}^{-1}$. In addition, the structured gold surfaces are robust and stable; thus we are able to clean and reuse them by cycling the potential from -0.4 to $1.6 \mathrm{~V}$ vs. SCE for 20 cycles in $10 \mathrm{mM}$ $\mathrm{H}_{2} \mathrm{SO}_{4}$ to remove the adsorbed thiol. We have stored and reused the same structured gold electrodes over a period of more than 22 weeks. These are significant advantages over the electrochemically roughened surface.

The SERS intensity at the structured gold surface increases linearly with laser light intensity (up to $3 \mathrm{~mW}$, the largest value accessible with our spectrometer) and depends upon the laser wavelength (for the sample used here the enhancement was largest for $785 \mathrm{~nm}$ excitation followed by 633 and then $514 \mathrm{~nm}$ ). The SERS intensity also varies with the film thickness and the void diameter indicating that the precise geometry of the structured gold film is an important factor. Table 2 reports the SERS intensity of the peak at $1571 \mathrm{~cm}^{-1}$ for films of different thicknesses prepared with templates of different sphere diameter. Taken together these results are consistent with EM enhancement of the SERS signal caused by the excitation of confined plasmons at the structured metal surface. This tentative explanation is consistent with our studies of the reflection spectra of these struc-

Table 2

Effect of film thickness and template sphere diameter on the SERS intensities for the benzene thiol peak at $1571 \mathrm{~cm}^{-1}$

\begin{tabular}{llc}
\hline $\begin{array}{l}\text { Template sphere } \\
\text { diameter }(\mathrm{nm})\end{array}$ & $\begin{array}{l}\text { Film thickness } \\
\text { with largest SERS } \\
\text { intensity }(\mathrm{nm})\end{array}$ & $\begin{array}{l}\text { SERS intensity } \\
\text { at } 1571 \mathrm{~cm}^{-1} \\
\text { (Raman counts) }\end{array}$ \\
\hline 450 & 132 & 6500 \\
500 & 398 & 15,833 \\
600 & 434 & 25,000 \\
700 & 138 & 8750 \\
800 & 645 & 16,600 \\
\hline
\end{tabular}

In each case, the film thickness is that which gives the most intense SERS under our experimental conditions. tured films $[18,22,23]$. Further work to confirm this is in progress.

The closest example in the literature to the work reported here is that of Tessier et al. [24] and Dick et al. [7]. Tessier et al. prepared inverse gold opals by the co-deposition of $25 \mathrm{~nm}$ gold particles and $630 \mathrm{~nm}$ latex microspheres to produce thick multilayer samples which they used as a SERS substrate. The crucial differences with our work are first that we are able to precisely control the topography of the film surface and this is important in determining the magnitude of the surface enhancement and second the use of gold nanoparticles, rather than electrodeposition, results in a film with high internal surface roughness. On the other hand Dick et al. used evaporation to deposit silver films over the top of close packed arrays of colloidal particles to produce a structured surface which showed good stability in in situ electrochemical experiments.

To investigate the use of the structured gold surface for electrochemical SERS, we recorded spectra for adsorbed pyridine as a function of the electrode potential, Fig. 3. The gold surfaces are stable under these conditions and give excellent SER spectra consistent with the published spectra for pyridine at silver electrode surfaces $[1,2,19]$ and the published work for pyridine on gold [4,25]. The two bands at 1010 and $1037 \mathrm{~cm}^{-1}$ have been assigned to symmetric ring breathing modes and occur at frequencies close to those for pyridine in aqueous solution. The band at $1026 \mathrm{~cm}^{-1}$ has been assigned to pyridine chemisorbed at the metal surface through the nitrogen lone pair. Between 0.2 and $-0.6 \mathrm{~V}$, as the potential is taken more negative the overall intensity of the band at $1010 \mathrm{~cm}^{-1}$ decreases whilst that at $1037 \mathrm{~cm}^{-1}$ remains approximately constant, neither

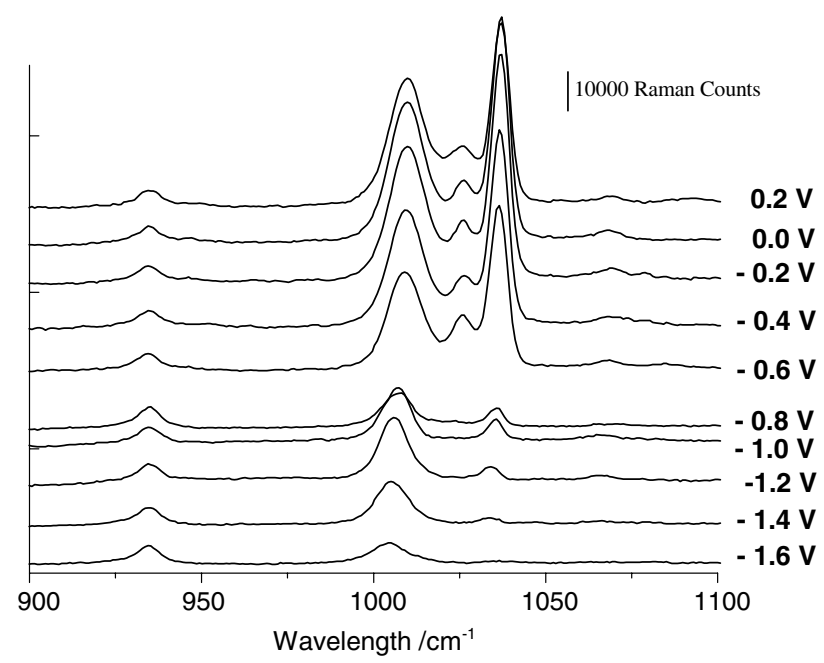

Fig. 3. Electrochemical SER spectra recorded in $0.1 \mathrm{M} \mathrm{KCl}$ containing $0.05 \mathrm{M}$ pyridine. The electrode was deposited through a $500 \mathrm{~nm}$ template and grown to $3 / 4$ diameter thickness. The spectra are offset for clarity. Spectra were recorded from a $5 \mu \mathrm{m}$ diameter spot on the electrode surface using $633 \mathrm{~nm} \mathrm{HeNe}$ laser, $3 \mathrm{~mW}$ power and an integration time of $60 \mathrm{~s}$ for 3 accumulations. 
band shifts significantly in position. However, between -0.6 and $-0.8 \mathrm{~V}$ there is a significant change in the appearance of the spectra - the bands at 1010 and $1037 \mathrm{~cm}^{-1}$ shift to lower wavenumber and are decreased in intensity; the band at $1026 \mathrm{~cm}^{-1}$, attributed to chemisorbtion of the pyridine at the gold surface, disappears. These changes are consistent with a change in the bonding of the pyridine to the gold surface and this behaviour follows that reported for pyridine on silver [1,2], although for the gold surface this occurs at a potential about $0.6 \mathrm{~V}$ negative of that on silver.

A recurrent problem which has hampered the application of SERS to electrochemistry over the last 30 years has been the irreversible loss in enhancement on cycling to high cathodic potentials. Although there have been some recent improvements in the stability of substrates [7] the situation is still far from ideal. As shown in Fig. 3, the SERS signal reduces dramatically at potentials below $-1.2 \mathrm{~V}$ and when the electrode was returned to more positive potentials the pyridine signals did not return to the same intensity. However, we attribute this to the formation of hydrogen in the pores at $-1.6 \mathrm{~V}$ since when the electrode was thoroughly wetted by a fresh solution of pyridine the SERS signal returned to its original intensity demonstrating that this substrate does not suffer an irreversible loss of SER activity under these conditions.

The Raman microscope was used in confocal mode to calculate the magnitude of the surface enhancement for pyridine at a structured and an electrochemically roughened gold surfaces, details of the method used can be found elsewhere [8]. The maximum surface enhancement factors obtained for the structured and roughened gold surfaces were $1.5 \times 10^{5}$ and $2.3 \times 10^{4}$, respectively.

\section{Conclusions}

We have shown that correctly structured gold surfaces formed by template electrodeposition can give strong surface enhanced Raman scattering. This enhancement is reproducible from place to place on the sample surface (unlike conventional roughened electrode surfaces) is stable and the surfaces can be cleaned and reused. We have also demonstrated the first use of these surfaces for in situ electrochemical SERS. Given the relative ease of preparation and their robust and reproducible nature, we believe that these surfaces have great promise for applications in electrochemical and analytical SERS.

\section{References}

[1] M. Fleischmann, P.J. Hendra, A.J. Mcquillan, Chem. Phys. Lett. 16 (1974) 163.

[2] D.L. Jeanmarie, R.P. Van Duyne, J. Electroanal. Chem. 84 (1977) 1.

[3] M.G. Albrecht, J.A. Creighton, J. Am. Chem. Soc. 99 (1977) 5215.

[4] A.G. Brolo, D.E. Irish, J. Lipkowski, J. Phys. Chem. B 101 (1997) 3906-3909.

[5] A. Kudelski, J. Bukowska, Vibrational Spectroscopy 10 (1996) 335-339.

[6] D.J. Rogers, S.D. Luck, D.E. Irish, D.A. Guzonas, G.F. Atkinson, J. Electroanal. Chem. 167 (1984) 237-249.

[7] L.A. Dick, A.D. Mcfarland, C.L. Haynes, R.P. Van Duyne, J. Phys. Chem. B 106 (2002) 853.

[8] Z.-Q. Tian, B. Ren, D.-Y. Wu, J. Phys. Chem. B 106 (2002) 9463.

[9] I. Baltog, N. Primeau, R. Reinish, J.L. Coutaz, Appl. Phys. Lett. 66 (1995) 1187.

[10] R.G. Freeman, K.C. Grabar, K.J. Allison, R.M. Bright, J.A. Davis, A.P. Guthrie, M.B. Hommer, M.A. Jackson, P.C. Smith, D.G. Walter, M.J. Natan, Science 267 (1995) 1629.

[11] M. Moskovits, Rev. Mod. Phys. 57 (1985) 783-826.

[12] P. Kambhampati, C.M. Child, M.C. Foster, A. Campion, J. Chem. Phys. 108 (1998) 5013-5026.

[13] G.C. Schatz, R.P. Van Duyne, Electromagnetic Mechanism of Surface Enhanced Spectroscopy, Wiley, New York, 2002.

[14] M.E. Abdelsalam, P.N. Bartlett, J. Baumberg, T. Kelf, S. Pelfrey, A.E. Russell, PCCP (submitted).

[15] M. Kawasaki, T. Sato, T. Yoshimoto, Langmuir 16 (2000) 5409 5417.

[16] G.W. Poehlein, R.H. Ottewill, J.W. Goodwin, Science and Technology of Polymer Colloids, Martinus Nijhoff Publishers, Boston, 1983.

[17] K. Nagayama, Colloids Surf. A 109 (1996) 363-374.

[18] P.N. Bartlett, J.J. Baumberg, S. Coyle, M.E. Abdelsalam, Faraday Discuss. 125 (2004) 117-132.

[19] X.M. Yang, K. Ajito, D.A. Tryk, K. Hashimoto, A. Fujishima, J. Phys. Chem. 100 (1996) 7293-7297.

[20] C.A. Szafranski, W. Tanner, P.E. Laibinis, R.L. Garrell, Langmuir 14 (1998) 3570.

[21] N.H. Kim, K. Kim, Chem. Phys. Lett. 393 (2004) 478.

[22] S. Coyle, M.C. Netti, J.J. Baumberg, M.A. Ghanem, P.R. Birkin, P.N. Bartlett, D.M. Whittaker, Phys. Rev. Lett. 8717 (2001), art. no. 176801 .

[23] M.C. Netti, S. Coyle, J.J. Baumberg, M.A. Ghanem, P.R. Birkin, P.N. Bartlett, D.M. Whittaker, Adv. Mater. 13 (2001) 1368 1370.

[24] P.M. Tessier, O.D. Velev, A.T. Kalambur, J.F. Rabolt, A.M. Lenhoff, E.W. Kaler, J. Am. Chem. Soc. 122 (2000) 9554.

[25] H. Yamada, Y. Yamamoto, Chem. Phys. Lett. 77 (1981) 520.

[26] S.W. Han, S.J. Lee, K. Kim, Langmuir 2001 (2001) 6981. 